\title{
Design Study of a Superconducting Gantry for Carbon Beam Therapy
}

\author{
J. Kim and M. Yoon* \\ Department of Physics, Pohang University of Science and Technology, \\ 77 Cheongam-Ro, Nam-Gu, Pohang, Gyeongbuk, Korea 790-784
}

(Received November 2015)

\begin{abstract}
This paper describes the design study of a gantry for a carbon beam. The designed gantry is compact such that its size is comparable to the size of the proton gantry. This is possible by introducing superconducting double helical coils for dipole magnets. The gantry optics is designed in such a way that it provides rotation-invariant optics and variable beam size as well as point-to-parallel scanning of a beam. For large-aperture magnet, three-dimensional magnetic field distribution is obtained by invoking a computer code, and a number of particles are tracked by integrating equations of motion numerically together with three-dimensional interpolation. The beam-shape distortion due to the fringe field is reduced to an acceptable level by optimizing the coil windings with the help of genetic algorithm. Higher-order transfer coefficients are calculated and shown to be reduced greatly with appropriate optimization of the coil windings.
\end{abstract}

PACS numbers: $29.20 . \mathrm{H}$

Keywords: Gantry, Beam Optics, Double helical coil

*Electronic address: moohyun@postech.ac.kr Fax: +82-54-279-3099 


\section{INTRODUCTION}

Treatment of malignant tumor by means of the ion-beam therapy (or equivalently ionbeam radiotherapy) has become increasingly popularized in recent decades. In the ionbeam therapy, an accelerated beam penetrates into the human body and deposits most of its energy into the DNA of tumor cells while mitigating the damage of normal tissues surrounding the tumor (Bragg peak). Compared to photons (or x-rays) ions have better dose-depth distribution.

Usually protons are used in the ion-beam therapy. At present about 50 proton-therapy facilities are in operation around the world and more than 105000 patients have been treated so far. But because of better physical and biological effect, heavier ions (usually ${ }^{12} \mathrm{C}^{6+}$ ions) are also employed. There are eight such facilities (six in Japan and two in Europe) active currently.

To deliver an ion beam from any direction to the tumor inside a patient, many ion-beam therapy facilities use gantries which are rotatable beam-transport systems mainly equipped with dipole magnets, quadrupoles, and scanning magnets togther with various diagnostic instruments. The gantry is in general massive and voluminous and so it is expensive to build. For example, the second proton gantry at Paul Scherrer Institut (PSI) in Switzerland is about $12 \mathrm{~m}$ wide and $4 \mathrm{~m}$ high and it's weight is approximately 100 tons.

The magnetic rigidity of the carbon ion is approximately 2.5 times higher than the proton, and so carbon ion requires higher energy than the proton energy does. For instance, for $30 \mathrm{~cm}$ penetration depth in water $430 \mathrm{MeV} / \mathrm{u}^{12} \mathrm{C}^{6+}$ is required whereas for proton only $220 \mathrm{MeV}$ is needed. The rigidity of $430 \mathrm{MeV} / \mathrm{u}^{12} \mathrm{C}^{6+}$ is $6.623 \mathrm{~T} \mathrm{~m}$. This implies that a gantry for carbon ion demands much larger space and heavier structure than proton gantry. For example, the space occupied by the carbon gantry in the Heidelberg Ion Therapy (HIT) facility is $22 \mathrm{~m}$ long and $14 \mathrm{~m}$ high [3] and the total weight of this gantry system is approximately $600 \mathrm{t}$ which is twice larger and five times heavier than the proton gantry in general. Obviously such a structure is not easy to build and operate.

Therefore, it is an important task to reduce the size of the carbon gantry. Various designs were reported so far.

In 2010, a preliminary study for design of a carbon gantry based on superconducting

toroidal combined-function magnet was reported [1]. This study confirmed that by employ- 
ing $5 \mathrm{~T}$ superconducting magnets the size of the carbon gantry could be reduced to proton gantry, approximately $10 \mathrm{~m}$ by $4 \mathrm{~m}$. The designed gantry is similar to the HIT design retaining all the beam optical features such as variable beam size from $4 \mathrm{~mm}$ to $10 \mathrm{~mm}$, point to parallel scanning capability, and rotational invariant optics. The scanning magnets cover $\pm 10 \mathrm{~cm}$ region in horizontal and vertical planes. In addition, our study reveals that the beam shape at the isocenter is significantly distorted due to nonlinear fields in the fringe field region of the large aperture $90^{\circ}$ dipole magnet. Sextuple components in the fringe field are found to be the cause of this problem.

To cure the beam shape distortion the coil winding in the main body of the dipole magnet has been redistributed in such a way that the resulting sextuple components in the main body compensate for the nonlinear field in the fringe field region. To find the optimized coil winding the genetic algorithm (GA) [2] has been invoked. Results indicated the compensation was made appropriately and subsequent preliminary particle tracking studies revealed to some extent the validity of the optimization of the GA.

This paper extends the previous studies and presents result of the further optimization of the coil winding. In Section II, we show a brief introduction of the beam optics of the designed gantry based on the superconducting magnet. This section is brief as mathematical result is already described elsewhere [3]. Section III describes the $90^{\circ}$ combined function dipole magnet. Section IV presents the method and results of the particle tracking. Finally, summary and conclusion is given in Section $\mathrm{V}$.

\section{GANTRY BEAM OPTICS}

The designed gantry is of similar type to the HIT carbon gantry [3]. Figure 1 shows the layout of the gantry which shows six quadrupole magnets, two $45^{\circ}$ dipole magnets, one $90^{\circ}$ dipole magnet, and two scanning magnets.

The six quadrupole magnets are used for beam size control at the isocenter in 4 - $10 \mathrm{~mm}$ range (for example, $R_{12}=4 \mathrm{~mm}$ and $R_{34}=4 \mathrm{~mm}$ ), dispersion-free optics at the isocenter $\left(R_{16}=R_{26}=0\right)$, and rotation invariant optics $\left(R_{11}=R_{33}=0\right)$. Here $R_{i j}$ is the $6 \times 6$ linear transfer matrix [4], $\mathbf{X}=R \mathbf{X}_{0}$ with $\mathbf{X}$ and $\mathbf{X}_{0}$ being 1 by 6 orbit vectors, $\mathbf{X}=$ 
$\left(x, x^{\prime}, y, y^{\prime}, l, \delta\right)^{T}$. The matrix equation is explicitly given by

$$
\left(\begin{array}{c}
x \\
x^{\prime} \\
y \\
y^{\prime} \\
l \\
\delta
\end{array}\right)=\left(\begin{array}{cccccc}
R_{11} & R_{12} & R_{13} & R_{14} & R_{15} & R_{16} \\
R_{21} & R_{22} & R_{23} & R_{24} & R_{25} & R_{26} \\
R_{31} & R_{32} & R_{33} & R_{34} & R_{35} & R_{36} \\
R_{41} & R_{42} & R_{43} & R_{44} & R_{45} & R_{46} \\
R_{51} & R_{52} & R_{53} & R_{54} & R_{55} & R_{56} \\
R_{61} & R_{62} & R_{63} & R_{64} & R_{65} & R_{66}
\end{array}\right)\left(\begin{array}{c}
x_{0} \\
x_{0}^{\prime} \\
y_{0} \\
y_{0}^{\prime} \\
l_{0} \\
\delta_{0}
\end{array}\right)
$$

Figure 2 shows the beam envelops along the beam path length for $\pm 90^{\circ}$ in $10^{\circ}$ step for 4 $\mathrm{mm}$ beam size at the iso center. It is seen that beam size at the isocenter does not depend on the rotation angle of the gantry. Also dispersion is shown to be localized between dipole magnets.

Upstream of the last $90^{\circ}$ combined-function dipole magnet two scanning magnets are placed, one for horizontal scanning and other for vertical scanning respectively. These magnets steer the beam by $\pm 10 \mathrm{~cm}$ in each plane. Locations of the scanning magnets were chosen to make point-to-parallel optics (i. e. $R_{22}=0$ from the position of the horizontal scanning magnet to the isocenter and $R_{44}=0$ from the vertical scanning magnet to the isocenter) to minimize the radiation power density of the patient's body. Thus in this design the source-to-axis distance (SAD) is infinity.

\section{III. $90^{\circ}$ BENDING MAGNET}

In Figure 1 the last magnet located upstream of the isocenter is a $90^{\circ}$ dipole magnet. This magnet is the most critical element in a carbon gantry because of its large aperture. The $90^{\circ}$ bending magnet requires combined magnetic multipole components in a body to satisfy the beam optics condition of the designed gantry and to correct high order effects.

Previously a novel winding concept was introduced for this magnet [1, 5-7]. Superposing two solenoid-like coils oppositely skewed with respect to a cylindrical axis $\cos \theta$ distribution of current density is achieved and the resulting magnetic field in the bore is a dipole. Coils wraps quater of torus shape and the beam is transported inside the wraped coils. The magnet can create not only dipole and quadrupole components but also sextupole and higher order multipoles. The winding follows the equation which is a function of the toroidal angle $\varphi$ 
and poloidal angle $\theta$ in toroidal coordinates (Figure 3). The winding equation for torus is given by

$$
\varphi=\frac{\theta}{n}+a_{0} \sin \theta+a_{1} \sin 2 \theta+a_{2} \sin 3 \theta+\cdots
$$

where $n$ is the number of windings in a full torus and $a_{0}, a_{1}, a_{2}, \ldots$ represent coefficients

of multipoles. Specifically, $a_{0}$ controls dipole field strength, $a_{1}$ controls quadrupole field strength, $a_{2}$ controls sextupole field strength, and so on.

Figure 4 shows three-dimensional view of the designed $90^{\circ}$ bending magnet. Strength of the magnetic dipole field is chosen to be $5.22 \mathrm{~T}$ at the center which makes $430 \mathrm{MeV}$ carbon ion be centered at $1.269 \mathrm{~m}$ in radius. Quadrupole field is set to have the field index of 0.5 to yield equal focussing in both planes but after design the position of the scanning magnets are readjusted from the calculation of the linear transfer matrix based on the multi-particle tracking.

Magnetic fields of the $90^{\circ}$ magnet was obtained by invoking a three-dimensional magnetic field solver OPERA-3D. Figure 5 shows the dipole field strength along the beam path of the $90^{\circ}$ bending magnet.

\section{PARTICLE TRACKING}

To verify the validity of the gantry design, particle tracking has been performed. For input beam parameters, $2 \mathrm{~mm} \times 0.5 \mathrm{mrad}$ horizontally, $10 \mathrm{~mm} \times 0.5 \mathrm{mrad}$ vertically, and momentum spread $0.2 \%$ were chosen, which is identical to the GSI design [3]. Total 1000 particle trajectories were obtained by numerically integrating the equations of motion in cylindrical coordinate system. During the integration, magnetic fields were obtained by interpolating the fields in the three dimensional space.

First, the reference particle was searched by tracing a particle at the center of the $90^{\circ}$ dipole magnet and particle energy was adjusted so that the resulting reference particle exits the magnet at $45^{\circ}$. Once the reference particle was found, transfer coefficients were obtained by tracking specially arranged particles. For integrating the equations of motion, the fourthorder Runge-Kutta integration method has been used. Positions of the scanning magnets were determined so as to yield the point-to-parallel optics. Then 1000 particles distributed as described in the above were tracked from the starting position at the entrance of the 
gantry to the position of the scanning magnets. At the scanning magnets kicks were applied in uniform steps and the particles were traced to the isocenter. In the calculation, the following parameters have been used: $R=1.269 \mathrm{~m}, \rho=0.186 \mathrm{~m}, n=864, a_{0}=0.162, a_{1}$ $=-0.00877018, a_{2}=-0.00098121$, and the current density $J=34287 \mathrm{~A} / \mathrm{m}^{2}$ with the coil radius of $0.5 \mathrm{~mm}$.

Figure 6 shows the kicks applied at the scanning magnets to span $\pm 10 \mathrm{~cm}$. Figure 7 shows the resulting beam distribution at the isocenter. It is seen that beam shapes are distorted significantly. Although kicks are applied in equal steps, resulting positions of the beam center at large kicks deviate from the linearity too much. Main cause of the shape distortions and the nonlinearity is found to be due to large sextupole components in the fringe field region of the $90^{\circ}$ magnet.

To cure the beam shape distortion, the coefficients $a_{i}$ have been readjusted by utilizing the genetic algorithm [2]. Biot-Savart law was used for calculation of the magnetic field due to coil windings. Final coil windings were then used for new magnetic field calculation by OPERA 3D code. Repeating the same procedure described above, the improved beam distribution at the isocenter is given in Figure 8. Significant improvement is achieved as this figure indicates. New multipole coefficients are $a_{0}=0.162, a_{1}=-0.00877018$, and $a_{2}$ $=0.000371945$.

For quantitative comparison, transfer coefficients were calculated [8]. The transfer coefficients are defined as

$$
\begin{aligned}
X_{i}=\sum_{j=1}^{6} R_{i j} X_{j 0}+\sum_{\substack{j, k \\
j \leq k}} T_{i j k} X_{j 0} X_{k 0}+ \\
\sum_{\substack{j, k, l \\
j \leq k \leq l}} U_{i j k l} X_{j 0} X_{k 0} X_{l 0}+ \\
\sum_{\substack{j, k, l, m \\
j \leq k \leq l \leq m}} W_{i j k l m} X_{j 0} X_{k 0} X_{l 0} X_{m 0}+\cdots
\end{aligned}
$$

where $R, T, U$, and $W$ are first-, second-, third-, and fourth-order transfer coefficients, respectively. $X_{i 0}$ and $X_{i}$ are the $i^{\text {th }}$ phase-space coordinates at the input and output positions, respectively.

Table I shows the comparison of the second-order transfer coefficients $T_{i j k}$ before and after optimization of the coil windings. It is seen that after adjustment of the coil winding the second-order coefficients have been reduced significantly. 
TABLE I: Comparison of $T_{i j k}\left(\mathrm{~cm} / \mathrm{mrad}^{2}, \mathrm{mrad} / \mathrm{mrad}^{2}\right)$ before and after the optimization of the coil windings

\begin{tabular}{lcc}
\hline \hline$T_{i j k}$ & Before improvement & After improvement \\
\hline$T_{122}$ & -3.263 & $1.627 \times 10^{-5}$ \\
$T_{144}$ & -3.249 & $-1.817 \times 10^{-4}$ \\
$T_{222}$ & -10.08 & $1.122 \times 10^{-4}$ \\
$T_{244}$ & -10.09 & $-5.026 \times 10^{-4}$ \\
$T_{322}$ & -18.61 & $4.557 \times 10^{-8}$ \\
$T_{344}$ & -18.61 & $1.170 \times 10^{-7}$ \\
$T_{422}$ & -2.793 & $1.623 \times 10^{-7}$ \\
$T_{444}$ & -2.796 & $4.524 \times 10^{-7}$ \\
$T_{124}$ & 3.246 & $5.444 \times 10^{-8}$ \\
$T_{224}$ & 10.09 & $3.983 \times 10^{-7}$ \\
$T_{324}$ & 18.61 & $-1.008 \times 10^{-4}$ \\
$T_{424}$ & 2.787 & $-1.098 \times 10^{-3}$ \\
\hline \hline
\end{tabular}

\section{CONCLUSIONS}

Beam optical studies for a gantry for carbon ion transport have been performed. The designed gantry is compact in the sense that its size is comparable to the size of existing proton gantries. Superconducting double helical coils were used for $90^{\circ}$ bending magnet. For this magnet, magnetic fields were calculated with the three-dimensional code and particles trajectories were calculated numerically by integrating equations of motion. We have found that there was a beam shape distortion at the isocenter which is large when the beam is steered at large angle. The distortion was found to be due to nonlinear fields in the fringe field region of the bending magnet of large aperture. We were able to demonstrate that the nonlinear distortion could be cured by means of adjusting coil winding distribution.

Our study needs to be extended by including an iron wrapped around the coils. The iron can shield the stray magnetic field as well as enhancing the magnetic field inside the bore. Design study for this case is in progress. 


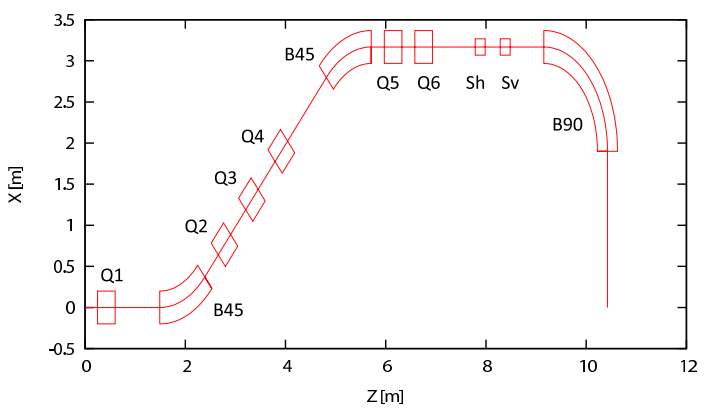

FIG. 1: Schematic layout for carbon gantry. Q1 Q6 are quadrupole magnets. B45 is a $45^{\circ}$ dipole magnet. Sh, Sv are horizontal steering magnet and vertical steering magnet respectively. B90 is a $90^{\circ}$ combined-function dipole magnet.

\section{Acknowledgments}

This work is supported by POSTECH Basic Science Research Institute Grant and the Heavy Ion Accelerator Project at Korea Institute of Radiological and Medical Science (KIRAMS).

[1] D.S. Robin, D. Arbelaez, S. Caspi, C. Sun, A. Sessler, W. Wan, and M. Yoon, Nucl. Instrum. Meth. Phys. Res. A 659484 (2011).

[2] K. Deb, Multi-objective Optimization using Evolutionary Algorithms (John Wiley and Sons, Ltd., West Sussex, England, 2001).

[3] M. Pavlovic, E. Griesmayer, R. Seemann, Nucl. Instrum. Meth. Phys. Res. A 545412 (2005).

[4] K. Brown, SLAC-75 (1982).

[5] R.B. Meinke, C.L. Goodzeit, M.J. Bell, IEEE Trans. Appl. Superconductivity 131369 (1003).

[6] S.Caspi, D.R.Dietderich, P.Ferracin, N.R.Finney, M.J.Fuery, S.A.Gourlay, A.R. Hafalia, IEEE Trans. Appl. Superconductivity 17 (part2) 2266 (2007).

[7] S.Caspi, M.Helm, L.J. Laslett,IEEE Trans. Magnetics 302419 (1994).

[8] M. Yoon and D. S. Robin, IEEE Trans. Nucl. Sci. 603837 (2013). 


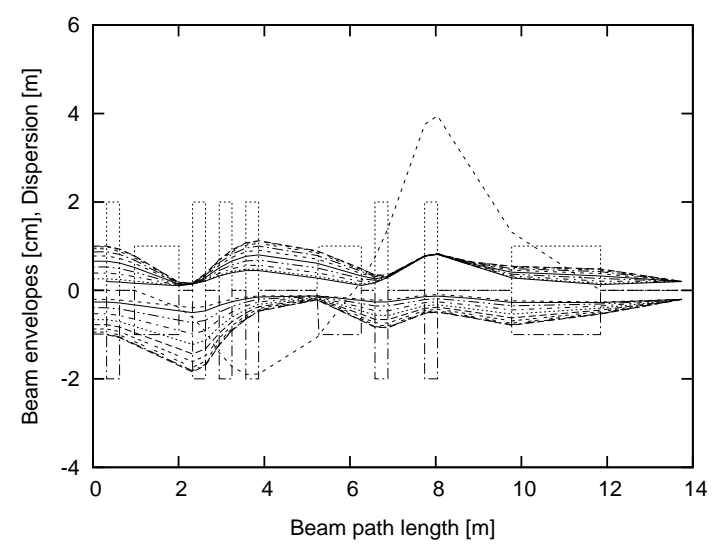

FIG. 2: Beam size variation along the beam path for $\pm 90^{\circ}$ in $10^{\circ}$ step. Dashed lines converging to a single point at the gantry end indicate beam envelopes for each rotation step. A single dashed line indicates horizontal dispersion function along the gantry.

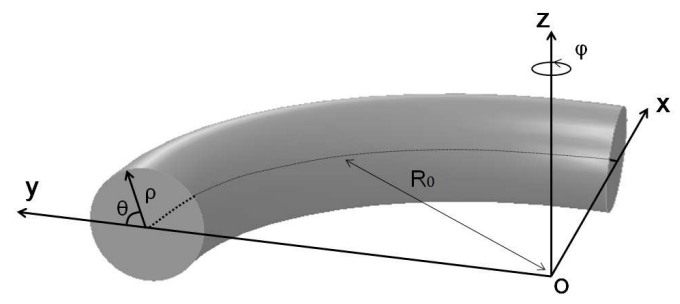

FIG. 3: Toroidal coordinates and cartesian coordinates: $R_{0}$ is the major radius of torus and $\rho$ is the minor radius of torus. $\varphi$ is the toroidal angle. $\theta$ is the poloidal angle. $O$ is the origin of the torus.

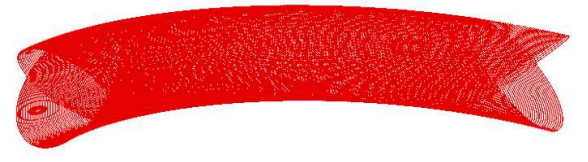

FIG. 4: $90^{\circ}$ bending magnet with double helical winding 


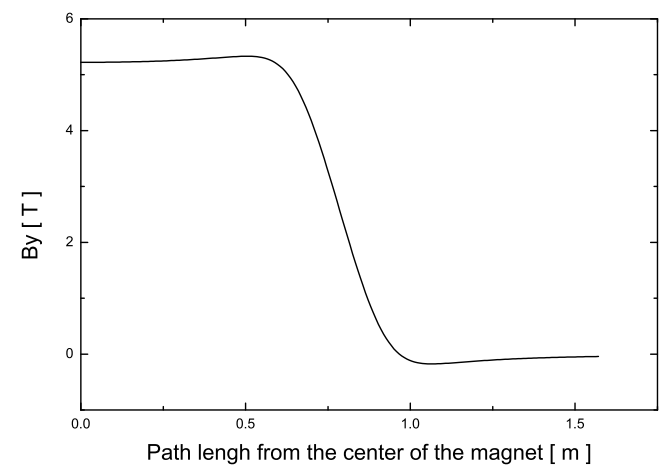

FIG. 5: Dipole field along the path length from the center of the $90^{\circ}$ bending magnet.

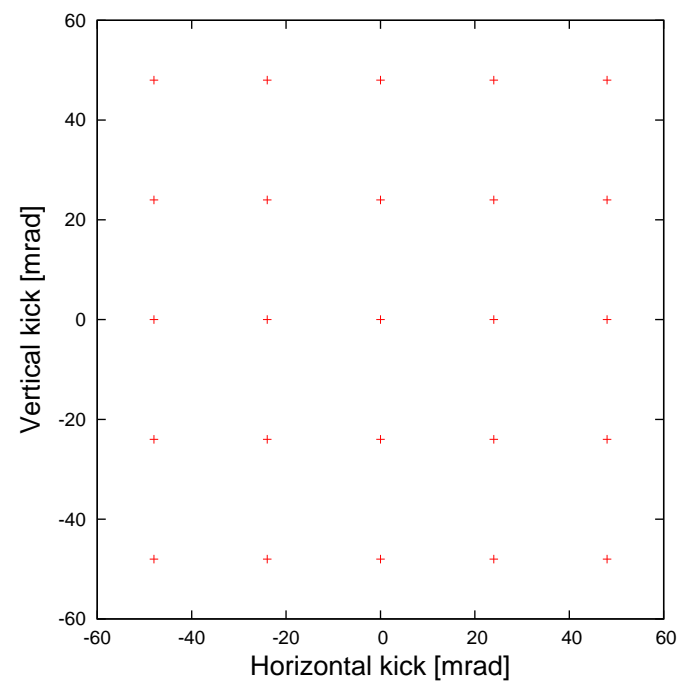

FIG. 6: Distribution of kick angles at horizontal and vertical scanning magnets

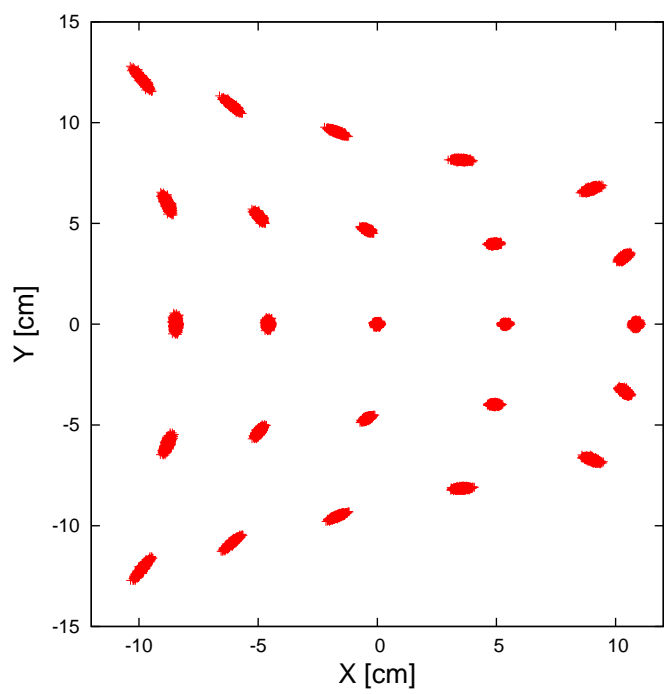

FIG. 7: Beam spots at the isocenter before optimization 


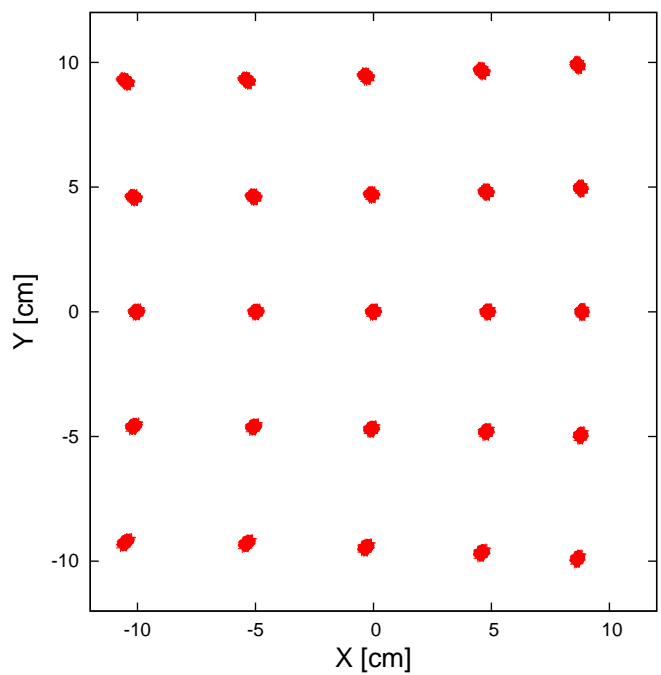

FIG. 8: Beam spots at the isocenter after readjusting coil winding distribution 